Rev. Biol. Neotrop. 3(2): 163-168. 2006

\title{
usÊnCIA de atividade mutagênica de Guazuma ulmifolia Lamb. (MUTAMBa) em CÉlULAS SOMÁtiCAS De DROSOPHILA MELANOGASTER
}

\author{
Cristiano José da Silva \\ Secretaria da Educação de Goiás, Goiânia, Goiás, Brasil, e-mail: cristianoprofeta@hotmail.com \\ Heleno Dias Ferreira \\ Laboratório de Sistema Vegetal, Departamento de Biologia Geral, Instituto de Ciências \\ Biolõgicas, Universidade Federal de Goiás, Campus II, 74001-970, Goiânia, Goiás, Brasil, \\ e-mail: heleno@icb.ufg.br
}

\section{Pedro Henrique Ferri}

Laboratório de Bioatividade Molecular, Instituto de Química, Universidade Federal de Goiás, Campus II, 74001-970, Goiânia, Goiás, Brasil, e-mail: heleno@icb.ufg.br

Wanderlene Blanco Nunes

Departamento de Biologia Geral, Instituto de Ciências Biológicas-I, Universidade Federal de Goiás, Campus II, 74001-970, Goiânia, Goiás, Brasil

Denise Gonçalves Pereira

Faculdade de Goiás - FAGO, Rua 67-A, n 216, Setor Norte Ferroviário, Goiânia, Goiás, Brasil

Salvador de Carvalho

Departamento de Biologia Geral, Instituto de Ciências Biológicas-I, Universidade Federal de Goiás, Campus II, 74001-970, Goiânia, Goiás, Brasil, e-mail: salvadorcarvalho2005@yahoo.com.br

Resumo: Guazuma ulmifolia Lamb., popularmente conhecida como mutamba, é uma planta medicinal encontrada no Cerrado brasileiro. O fitoterápico mutamba é usado na medicina alternativa para o tratamento de: diarréia, asma, bronquite, febre, elefantíase, sífilis, obesidade, hanseníase, queda de cabelos, disenteria, entre outros. Também é considerado adstringente, depurativo e sudorífero. O presente trabalho avaliou o efeito genotóxico da mutamba, através do teste SMART/asa, em larvas de terceiro estágio de desenvolvimento embrionário de Drosophila melanogaster descendentes do cruzamento padrão (ST) e do cruzamento de alta atividade metabólica (HB). Pelos resultados não foram observadas alterações estatisticamente significativas nas freqüências de manchas entre as diferentes concentrações do fitoterápico (500, 50, 35 e $25 \mathrm{mg} / \mathrm{mL}$ ) e o controle negativo nos descendentes de nenhum cruzamento, indicando que nestas condições experimentais G. ulmifolia não apresentou efeito genotóxico em células somáticas de $D$. melanogaster.

Palavras-chave: citotóxico, genotóxico, mutamba, Guazuma, Malvacese.

Abstract: Guazuma ulmifolia Lamb. is a medicinal plant, locally called "mutamba", which grows in the Cerrado of Brazil. Mutamba phytotherapic is used in alternative medicine to treat diarrhea, asthma, bronchitis, fever, elephantiasis, syphilis, obesity, leprosy, hairlass, dysentery, among others. This phytothergric agent is also used as astringent, depurative, and sudorific. This study evaluated the genotoxic effect of the mutamba through somatic mutation and recombination test (SMART/wing) in the third instar of Drosophila melanogaster larvae resulting from the standard cross (ST) and high bioactivation cross (HB). There were no statistically significant alterations in the frequencies of spot among different concentrations of the phytoterapic $(500,50,35,25 \mathrm{mg} / \mathrm{mL})$ to the negative control for the descendents of all crosses. This suggests that $G$. ulmifolia had no genotoxic effects in somatic cells of $D$. melanogaster in these conditions.

Key Words: citotoxic, genotoxic, mutamba, Guazuma, Malvacese.

\section{INTRODUÇÃO}

1 utilização de plantas medicinais é o resultado do acúmulo secular de conhecimentos empíricos sobre a ação dos vegetais por diversos grupos étnicos. Entretanto existem questões pertinentes à padronização de técnicas de produção e comercialização de fitoterápicos (Di Stasi, 1996). 
Esta preocupação levou a Organização Mundial de Saúde, em 1978, a estabelecer um programa mundial com o objetivo de avaliar e padronizar os métodos utilizados em medicina natural (Campelo, 1983).

O consumo indiscriminado de plantas medicinais, muitas delas tóxicas, pode trazer riscos à saúde, pois, assim como para os produtos alopáticos, também existe uma dosagem limiar para cada tipo de erva medicinal. Como conseqüência do uso inadequado, podem ocorrer desde intoxicações a eventos mutacionais em tecidos somáticos e germinativos, que podem levar ao desenvolvimento de doenças somáticas, efeitos teratogênicos e danos genéticos hereditários (D`Oliveira, 1998; Wong, 2003).

Diversas ervas e chás ricos em alcalóides pirrolizidínicos, comumente usados em medicina popular ou na dieta, apresentam atividades genotóxicas (Ames, 1983; Tresvenzol et al., 2006).

A grande maioria dos carcinógenos na verdade inicia sua atividade tumoral através de interações com o DNA, levando a lesões genéticas permanentes, que se expressam como mutações gênicas e/ou aberrações cromossômicas, envolvendo o ciclo celular (Bronzetti et al., 1996; Gonzalgo \& Jones, 1997; Pereira, 1999; Reis et al, 2002; Andrade, 2004).

A casca e a entrecasca de Guazuma ulmifolia (mutamba) é rica em componentes com propriedades farmacológicas. Paralelamente esses princípios isolados têm sido correlacionados com o tratamento de diversas doenças. Assim o ß-sitosterol atua contra as lipoproteinemias; os triterpenos são usados como anti-inflamatórios (pneumonia e bronquite); a cafeína atua como diurético e estimulante do SNC e dos músculos cardíacos; os alcalóides são tidos como anti-microbianos, analgésicos, anti-espasmódicos e estimulantes do SNC, os taninos são excelentes no combate aos processos de disenteria (Windholz, 1983; Almeida et al., 1998; Rizzo et al., 1990; Rizzo et al. 1999; Tridente, 2002;

Neste trabalho foram analisados os possíveis efeitos genotóxicos da planta G. ulmifolia (mutamba) através do teste SMART/asa com $D$. melanogaster, uma vez que o uso desta planta é expressivo no Estado de Goiás.

\section{Material e métodos}

Casca e entrecasca da planta G. ulmifolia foram coletadas no município de São Luís de Montes Belos, Estado de Goiás, Brasil; em seguida, foram trituradas e usadas na preparação de infusão nas concentrações recomendadas para uso humano, ou seja, 1 - 2 g do pulverizado diluído em $30 \mathrm{~mL}$ de água.
Para a realização dos experimentos foram estabelecidas as concentrações de 25,35 , 50 e $500 \mathrm{mg} / \mathrm{mL}$, preparadas no momento do tratamento.

Para o controle positivo, utilizou-se uretano (etil carbamato), por ser genotóxico conhecido, dissolvido em tampão fosfato $0,03 \mathrm{M}$, em pH 6,8 (Merck, Darmstd) na concentração de $20 \mathrm{mM}$, também preparado na hora do tratamento. O controle negativo foi feito com água destilada esterilizada.

Para manter as linhagens de D. melanogaster, bem como para a realização dos cruzamentos das linhagens, utilizou-se o meio de cultura banana-ágar (Marques et al., 1996) modificado, distribuído em garrafas estéreis de $1 / 4$ de litro (Graf et al., 1984).

O meio de cultura para obtenção de larvas constituiu-se de uma base sólida feita com ágar a 2,5\% $(0,5 \mathrm{~cm})$, coberta com uma camada de fermento biológico mais açúcar (Graf et al.,1984).

Para o tratamento crônico das larvas de terceiro estágio (com $72 \pm 4$ horas), utilizou-se o meio de cultura sintético (fórmula 4-24, Carolina Biological Supply Company, Burlington, North Carolina).

Três linhagens diferentes de D. melanogaster foram usadas nos cruzamentos: machos da linhagem marcadora designada mwh (multiple wing hair) e fêmeas virgens designadas flr $^{3}$ (flare) e ORR; flr ${ }^{3}$.

Para a avaliação desse teste utilizaram-se dois tipos de cruzamentos:

1 cruzamento padrão (ST);

2 cruzamento de alta bioativação (HB).

Machos da linhagem mwh, com 2-3 dias de vida, foram cruzados com fêmeas virgens da linhagem marcadora $\mathrm{flr}^{3}$ (cruzamento ST) ou ORR; flr ${ }^{3}$ (cruzamento HB). Por volta de 72 \pm 4 horas após o início da ovoposição, as larvas de terceiro estágio foram coletadas e colocadas em tubos de fundo chato contendo $1,5 \mathrm{~g}$ de meio sintético, sendo adicionados $5 \mathrm{~mL}$ das diferentes soluções de tratamento:

- Controle negativo: água destilada estéril;

- Controle positivo: uretano (20 mM);

- C1: fitoterápico puro $(500 \mathrm{mg} / \mathrm{mL})$;

- C2: fitoterápico $(50 \mathrm{mg} / \mathrm{mL})$;

- C3: fitoterápico $(35 \mathrm{mg} / \mathrm{mL})$;

- C4: fitoterápico (25 mg/mL).

Foram realizadas 3 repetições por dose. As larvas permaneceram à temperatura de $25^{\circ} \mathrm{C}$, com umidade relativa de $60 \%$, até atingir o estágio de imago. 
As asas de cinco moscas do sexo feminino e cinco do sexo masculino foram separadas do corpo, com o auxílio de pinças de relojoeiro, distendidas sobre uma lâmina de vidro e analisadas em micros-cópio com 400 x de ampliação (Graf et al., 1984; Frei \& Würgler, 1988; 1995).

Os marcadores genéticos expressos alteram a diferenciação final dos pêlos, podendo apresentar o fenótipo mwh (multiple wing hairs) - pêlos múltiplos, ou o fenótipo flr ${ }^{3}$ (flare) - pêlos em forma de chama. A linhagem celular derivada de uma célula genotipicamente alterada forma um clone que é reconhecido, no adulto, como uma mancha (spot). Essas manchas podem ser simples, quando apresentam somente pêlos mwh ou flr ${ }^{3}$, ou gêmeas, quando apresentam pêlos mwh e flr ${ }^{3}$.

A análise estatística foi feita conforme os dados relativos aos tratamentos e os respectivos controles negativos e positivos. Quando existe a presença de manchas em número considerável tem-se o elemento estatístico fundamental para a inferência.

O procedimento utilizado se baseia em duas hipóteses:

(i) Ho: A freqüência de mutação espontânea e induzida nas moscas tratadas não é maior que a freqüência de mutação no respectivo controle.

(ii) Ha: A freqüência de mutação induzida nas moscas tratadas não é menor que $m$ vezes a maior freqüência de mutação espontânea observada no controle.

Para testar as hipóteses foi aplicado o teste binomial condicional (Kastenbaum \& Bowman, 1970) ou teste Qui-quadrado para proporções (Frei \& Wurgler, 1988). Esse teste foi usado com níveis de significância $a=b=0,05$. Nesse caso, podem ser consideradas as seguintes decisões:

(i) Aceitam-se ambas as hipóteses e, como elas não podem ser verdadeiras simultaneamente, nenhuma decisão pode ser tomada, sendo, assim, o resultado inconclusivo.

(ii) Aceita-se a primeira hipótese e rejeita-se a segunda, obtendo-se o resultado negativo.

(iii) Rejeita-se a primeira hipótese e aceitase a segunda e o resultado será positivo.

(iv) Rejeitam-se ambas as hipóteses e o resultado é um fraco-positivo.

$\mathrm{O}$ valor de $\mathrm{m}$ é fixado em 2 , devido à alta freqüência de mutação espontânea verificada em manchas simples pequenas e o total de manchas simples. Para a análise estatística, as manchas foram agrupadas da seguinte forma: 1 -Manchas simples pequenas (MSP), de uma a duas células $\left(\mathrm{mwh}\right.$ ou $\left.\mathrm{flr}^{3}\right) ; 2$ - Manchas simples grandes (MSG), com três ou mais células (mwh ou $\mathrm{flr}^{3}$ ) e 3 - Manchas gêmeas (MG) com áreas mwh e $\mathrm{flr}^{3}$. Os três tipos de manchas foram avaliados separadamente, conforme mostram as Figuras 1 e 2 e os resultados.

\section{Resultados e discussão}

Conforme as Figuras 1 e 2, os cruzamentos padrão (ST) e de alta bioatividade (HB), a mutamba (G. ulmifolia) nas concentrações de 500, 50, 35 e $25 \mathrm{mg} / \mathrm{mL}$, não induziu aumento estatisticamente signi-ficativo (á=â=0,05) no número de manchas pequenas simples (1-2 células), grandes simples ( $>3$ células), gêmeas e no total de manchas, quando comparado com o controle negativo, usando água destilada estéril.

O controle positivo (uretano) induziu aumento estatisticamente significativo (á $=\hat{a}=0,05$ ) no número de manchas pequenas simples (1-2 células), grandes simples ( $>3$ células), gêmeas e no total de manchas, quando comparado com o controle negativo. Esses resultados confirmam a validade do teste, assegurando ausência de efeitos genotóxicos da planta G. ulmifolia, em células somáticas de $D$. melanogaster.

Diversos princípios ativos, tais como bsitosterol, cafeína e tanino, encontrados em G. ulmifolia apresentaram efeitos antimutagênicos devido a interações com radicais livres, conferindo proteção contra danos oxidativos (Chung et al., 1998; Park et al., 2003; Piosik et al., 2003; Villasenor et al., 2002; http://www.raintree.com/ mutamba.htm; http://www.iarc.fr).

Algumas pesquisas com mutamba evidenciaram efeito antioxidante da casca e da folha devido à presença de pro-antocianidina, sinalizando para a possibilidade de seu uso na profilaxia de danos ao material genético (Park et al., 2003; Piosik et al., 2003). De acordo com Fragiorge (2000), as atividades moduladoras são importantes na redução da incidência de mutações causadas pelos quimioterápicos antineoplásicos, especialmente os geradores de radicais livres.

A ausência de atividade mutagênica da mutamba pode estar relacionada com a presença de princípios ativos que atuam como antioxidantes, neutralizando a ação de outros compostos sem essa atividade moduladora (Fragiorge, 2000). 


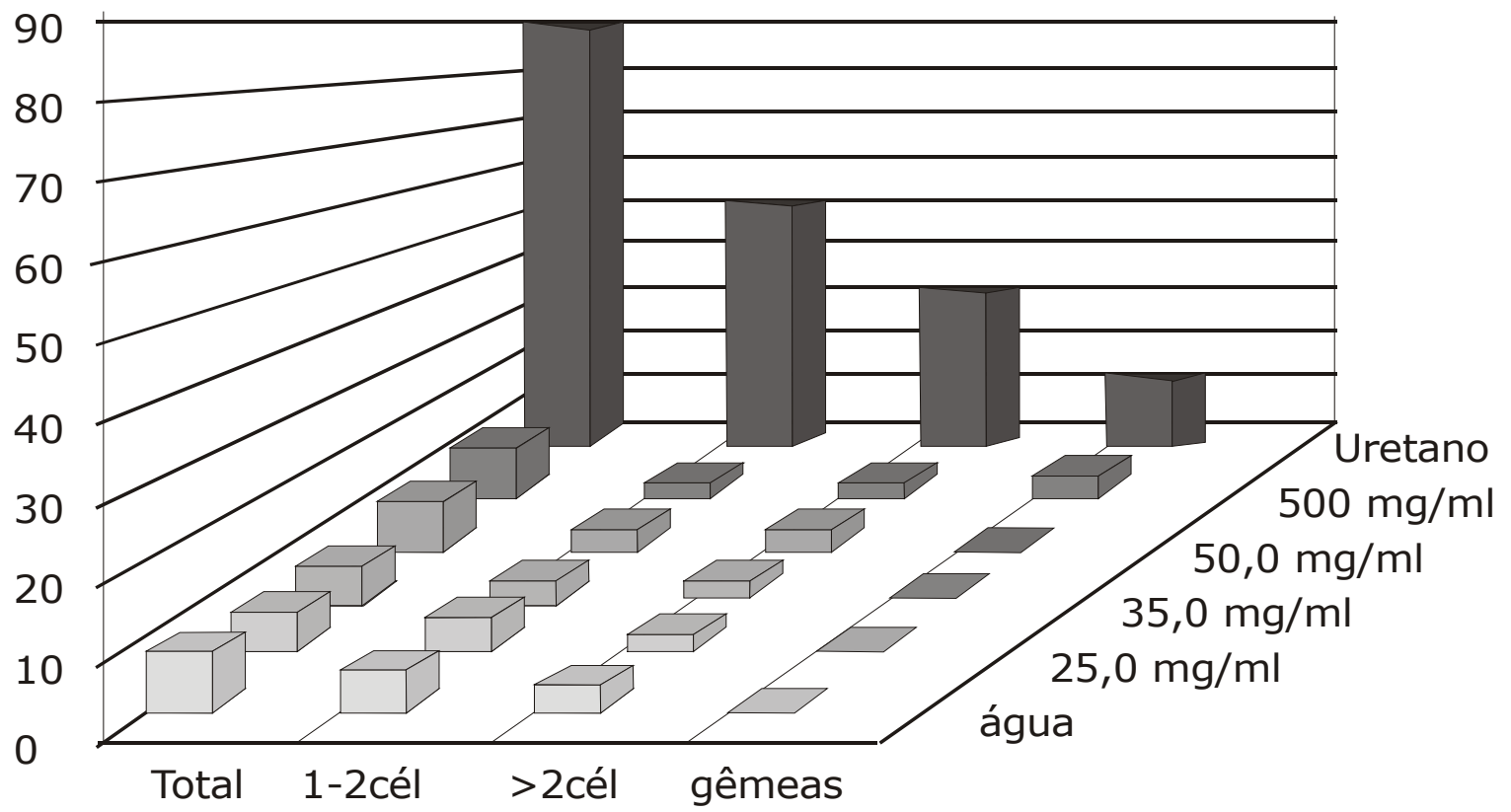

Figura 1 - Distribuição de manchas de acordo com o número de células mutantes em asas de D. melanogaster (40 moscas por tratamento) descendentes de cruzamento padrão (ST), tratadas com extrato aquoso de G. ulmifolia, água estéril (controle negativo) e uretano $20 \mathrm{mM}$ (controle positivo).

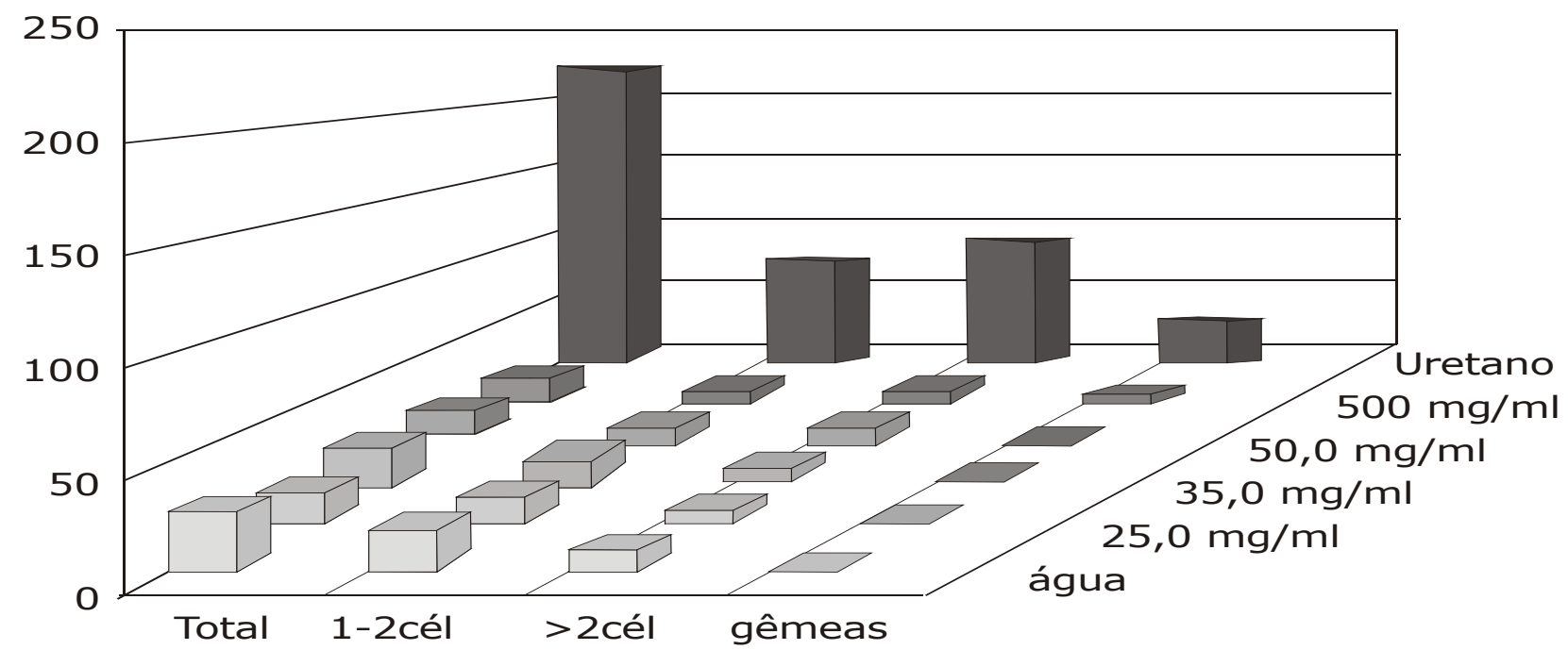

Figura 2 - Distribuição de manchas de acordo com o número de células mutantes em asas de D. melanogaster (40 moscas por tratamento) descendentes de cruzamento de alta capacidade, bioativação (HB), tratadas com extrato aquoso de G. ulmifolia, água estéril(controle negativo) e uretano 20mM (controle positivo). 


\section{Conclusão}

Considerando D. melanogaster como organismo teste e as condições experimentais descritas, pode-se concluir que:

1 - O extrato de mutamba não exerceu atividade do tipo mutação de ponto, deleção, não-disjunção ou recombinação mitótica em células somáticas de larvas de D. melanogaster de terceiro estágio oriundas do cruzamento padrão (ST), sugerindo que o fitoterápico não é um agente genotóxico de ação direta.

2 - O extrato de mutamba também não foi efetivo na indução de eventos de mutação e/ou recombinação mitótica em células somáticas de larvas de terceiro estágio oriundas do cruzamento de alta atividade metabólica (HB), indicando que o fitoterápico não é pró-mutágeno, pois não demonstrou ser agente genotóxico de ação indireta.

A presença de compostos na mutamba que atuam protegendo o DNA de danos causados por xenobióticos sinaliza possível efeito modulador desse fitoterápico.

Com base nestes resultados, recomendase avaliar a mutamba quanto ao seu potencial modulador de mutações em larvas de terceiro estágio de $D$. melanogaster.

\section{ReferênCIAS}

Almeida, S. P., C. E. B. Proença, S. M. Sano \& J. F. Ribeiro. 1998. Cerrado: espécies vegetais úteis. Planaltina, EMBRAPA, CPAC.

Ames, B. N. 1983. Dietery carcinogens and anticarcinogens. Science 221: 1256-1264.

Andrade, A. N. 2004. Micotoxinas: importância na alimentação (uma revisão). Rev. Bras. Canc. 50: 139-175.

Bronzetti, G., C. Della Croce., P. Arentini \& R. Fiorio. 1996. Antimutagenesis and anticancer effects. J. Environ. Pathol. Toxicol. Oncol. 15: 59-64.

Campelo, C. R. 1983. Plantas medicinais de Pernambuco I. VII Reunião Nordestina de Botânica, São Luís.

Chung, K.T., T.Y. Wong, C.I. Wei, Y.W. Huang \& Y. Lin. 1998. Tannins and human health: a review. Crit. Rev. Food. Sci. Nutr. 38: 421-64.
Di Stasi, L. C. 1996. Plantas medicinais: arte e ciência. Um guia de estudo interdisciplinar. São Paulo, Editora da Universidade Estadual Paulista.

D’Oliveira, M. I. P. 1998. Avaliação da atividade genotóxica e mutagênica do barbatimão (Stryphinodendron adstringens Mart.) em cepas bacterianas. Dissertação de Mestrado.

Fragiorge, E. J. 2000. Efeitos moduladores de ácido ascórbico quando associado ao cloridrato de doxorrubicina em células somáticas de Drosophila melanogaster, tratadas na presença e ausência de luz. Dissertação de Mestrado. Universidade Federal de Uberlândia, Uberlândia, MG.

Frei, H. \& F. E. Würgler. 1995. Optimal experimental design and sample size for the statistical evaluation of data from somatic mutation and recombination tests (SMART) in Drosophila. Mutat. Res. 334: 247-358.

Frei, H. \& F. E. Würgler. 1988. Statistical methods to decide whether mutagenicity fest data from Drosophila assay indicate a positive, negative, or inconclusive result. Mutat. Res. 203: 297-308.

Gonzalgo, M. L. \& P. A. Jones, 1997. Mutagenic and epigenetic effects of DNA methylation. Mutat. Res. 386: 107-118.

Graf, U., F.E. Katz, A.J. Frei, H. Juon, C.B. Hall \& P.G. Kale. 1984. Somatic mutation and recombination test in Drosophila melanogaster. Environ. Mutagen. 6: 153-88.

Graf, U., K. A. Suresh, G.R Judith \& E. W. Friedrich 1998. Antigenotoxicity studies in Drosophila melanogaster. Mutat. Res. 18: 203-209.

International Agency for Research on Cancer - IARC's Mission: Cancer research for cancer control. Disponível em: <http://www. iarc.fr.>. Acesso em nov./2005.

Kastenbaum, M. A. \& K. O. Bowman. 1970. Tables for determining the statitstical significance of mutation frequences. Mutation Res., 9: 527-549.

Marques, E. K., M. Napp, H. Winge \& A. R. Cordeiro. 1966. A corn meal, soybean flour, wheat germ medium for Drosophila. Dros. Inform. Serv. 41: 187. 
Park, K.Y., K.O. Jung., S.H. Rhee \& Y.H. Choi. 2003. Antimutagenic effects of doenjang (Korean fermented soypaste) and its active compounds. Mutat. Res. 523-524: 43-53.

Pereira, D. G. 1999. Avaliação do potencial mutagênico e/ou recombinogênico de Hyptidendron canun, em células germi-nativas e somáticas de Drosophila melanogaster. Dissertação de Mestrado. Instituto de Ciências Biológicas, Universidade Federal de Goiás, Goiânia.

Piosik, J., K. Ulanowska., A. Gwizdek-Wisniewska., A. Czyz., J. Kapuscinski \& G. Wegrzyn. 2003. Evaluation of mutagenic effects of polycyclic aromatic agents (quinacrine mustard, ICR-191 and ICR-170) by caffeine and pentoxifylline. Mutat. Res. 530(1-2): 47-57.

Raintree Nutrition/Tropical Plant Database Mutamba (Guazuma ulmifolia), disponível em: $<$ http://www. rain-tree.com/mutamba. $\mathrm{htm}>$. Acesso em nov./2005

Rizzo, J. A., M.S.R. Monteiro \& C. Bittencourt. 1990. Utilização de plantas medicinais em Goiânia. In: Anais do XXXVI Congresso Brasileiro de Botânica. v. 2: 691-707. Curitiba, Paraná, Brasil.

Rizzo, J.A., I.F.P. Campos, M.C. Jaime, T. Munhoz \& W.F. Morgado. 1999. utilização de plantas medicinais nas cidades de Goiás e Pirenópolis, Estado de Goiás. Rev. Ciênc. Farm. 20: 431-447.
Reis, S. R. A., M. Sadigursky, M. G. S. Andrade, L. P. Soares, A. R. Espírito Santo \& D. S. V. Boas. 2002. Efeito genotóxico do etanol em células da mucosa bucal. Pesq. Odontol. Bras. 16: 221-225.

Tresvenzol, L. M., J. R. PAULA, A. F. Ricardo, H. D. Ferreira \& D. T. Zatta. 2006. Estudo sobre o comércio informal de plantas medicinais em Goiânia e cidades vizinhas. Rev. Eletr. Farm. 3: 23-28.

Tridente, R. D. 2002. O uso de plantas medicinais na cidade de Porangatu, Estado de Goiás. Dissertação de Mestrado. Instituto de Ciências Biológicas, Universidade Federal de Goiás, Goiânia.

Villasenor I. M., J. Angelada., A.P. Canlas \& D. Echegoyen. 2002. Bioactivity studies on beta-sitosterol and its glucoside. Phytother. Res. 16: 417-21.

Windholz, M. 1983. The Merck index: an encyclopedia of chemicals, drugs and biologicals. 10th ed., New Jersey, Merck \& co. N. J., USA.

Wong, A. 2003. Os usos inadequados e os efeitos adversos de medicamentos na prática clínica. J. Pediatr. 79:379-380.

Recebido em 17.III.2006 Aceito em 30.XI.2006 